\title{
Control político indirecto mediante la acción de tutela, desde la perspectiva de la sentencia t-760 de 2008*
}

\author{
Indirect political control through guardianship (legal tutela), from the \\ perspective of judgment t-760 of 2008
}

\author{
Oscar Mauricio Vargas Quintero** \\ Felipe Beltrán EsPinosA ${ }^{* * *}$
}

\begin{abstract}
* El presente trabajo es resultado de la investigación "Mecanismos de Control y Participación Social: Estrategias para la Paz. Estudio de caso en el municipio de Zipaquirá", liderado desde la Institución Universitaria Colegios de Colombia, UNICOC, con participación del Semillero de investigación del Colegio Jurídico de UNICOC

** Abogado,. Integrante de Semillero de investigación del Colegio Jurídico de UNICOC. E mail: ovargas@unicoc.edu.co

***Abogado, Integrante de Semillero de investigación del Colegio Jurídico de UNICOC. E mail: fbeltrane@unicoc.edu.co
\end{abstract}

Fecha de recepción: Marzo de 2019

Fecha de aprobación: julio de 2019

Para citar este artículo / To reference this article Vargas, O.M., Beltrán, F. (2019) Control político indirecto mediante la acción de tutela, desde la perspectiva de la sentencia t- 760 de 2008. Inciso, 21; 103-127.

DOI: http://dx.doi.org/10.18634/incj.21v.1i.914

\section{Resumen}

El artículo tiene como finalidad sugerir la manera de configurar un control político a las actuaciones u omisiones de las entidades del Estado, a través del uso de los mecanismos Constitucionales de protección de derechos por parte de la ciudadanía. En ese orden de ideas, a través de una metodología analítico-descriptiva, se establecen los elementos necesarios para la educación y pedagogía en competencias ciudadanas desde la universidad, siendo una alternativa en el proceso de apropiación de la participación en el control político, como se demuestra a manera de ejemplo en las implicaciones de la expedición de la sentencia T-760 de 2008 frente a la necesidad de empoderar a las personas en la defensa de sus derechos y cómo esta participación genera cambios estructurales en las instituciones del Estado, en pro de la garantía de los Derechos Fundamentales.

Palabras clave: formación política, política pública, pedagogía, educación y ciudadanía. 


\begin{abstract}
The purpose of this article is to suggest how to configure a political control to the actions or omissions of the State entities, through the use of the Constitutional mechanisms of protection of rights by the citizens. This way, through an analytical-descriptive methodology, there are established the necessary elements for education and pedagogy in citizen competencies from the university, being an alternative in the process of appropriation of participation in political control, as demonstrated by way of example in the implications of the issuance of judgment T-760 of 2008, regarding the need to empower people in the defense of their rights; and how this participation generates structural changes in state institutions, in favor of guaranteeing Fundamental Rights.
\end{abstract}

Keywords: Political formation, public policy, pedagogy, education and citizenship.

\title{
INTRODUCCIÓN
}

Se plantea la necesidad de fortalecer la pedagogía y la educación ciudadana en los mecanismos constitucionales de protección de derechos, por medio del cual se puede generar control político y políticas públicas, en procura de exigir de las entidades públicas el cumplimiento de los mandatos legales y constitucionales en cuanto al cumplimiento de las funciones que les fueron asignadas.

Teniendo en cuenta que el objetivo de esta investigación se basó en resaltar la importancia del control político a partir del uso de los mecanismos constitucionales, este trabajo está desarrollado en tres secciones importantes mediante una investigación analítica descriptiva, en cuanto al desarrollo a modo de ejemplo del contexto histórico legal de la salud en Colombia, lo que permitió entender la coyuntura en la que se enmarcó la decisión de la Corte Constitucional en la sentencia T-760 de 2008 y también un componente analítico en el cual se constata la coexistencia de factores que impactan en la participación ciudadana activa frente a los mecanismos constitucionales y cómo deben confluir estos factores en la apropiación de conocimientos para ampliar dicha participación.

Es así que la primera sección, hace referencia a la enunciación de las acciones constitucionales como mecanismos de protección de derechos, también se realiza la aproximación conceptual de los elementos necesarios para entender y ejercer control político y generar políticas públicas por parte de la ciudadanía. La segunda sección, se enfoca en el análisis de la sentencia T-760 de 2008 proferida por la Corte Constitucional, específicamente frente al control político y la generación de parámetros mínimos de políticas públicas en lo que respecta al enfoque prestacional de los derechos fundamentales, como un ejemplo del impacto de la participación de la ciudadanía a través del mecanismo constitucional como fue la acción de tutela, la cual en principio no tiene esta función principal, sin embargo, para el área de la salud en Colombia resulta 
ser definitiva. La última sección plantea la alternativa de la importancia de la educación en competencias ciudadanas como una herramienta para fortalecer la participación y el empoderamiento político de la ciudadanía por medio del ejercicio de las acciones constitucionales, donde se toma la experiencia del Diplomado en Competencias Ciudadanas, liderado por la Institución Universitaria Colegios de Colombia-UNICOC- que contó con la participación activa del semillero de investigación del Colegio Jurídico de la misma institución, desarrollado en el municipio de Zipaquirá, en el cual se brindaron las bases y fundamentos a líderes sociales para lograr un acceso eficaz y efectivo a los mecanismos de protección de derechos y del cual se extraen sus resultados, como evidencia de la pertinencia que tiene este tipo de iniciativa en procura de lograr una sociedad democráticamente activa.

Bajo esta línea de ideas, además, se presenta este resultado de investigación a la comunidad académica, para invitar a que los procesos de construcción y réplica del conocimiento sean un eje que facilite la pedagogía en la apropiación de las competencias ciudadanas por parte de la ciudadanía.

Lo anterior, adquiere relevancia en razón a la falta de aplicación y desarrollo de las leyes, proyectos, políticas y programas, que en definitiva afectan a la sociedad como se evidencia en el desarrollo de este trabajo específicamente en el contexto del derecho a la salud en Colombia. Esto último, sirve de ejemplo de las falencias legales que llevan a la intervención de la Corte Constitucional en la protección de los derechos individuales de los accionantes, así como en la realización de un control a las actuaciones y omisiones del ejecutivo y la formulación de parámetros mínimos con que deben contar las políticas públicas; lo cual es una muestra del significado de la participación activa de las personas en el control político a través del uso de los mecanismos constitucionales, como fue la acción de tutela, de iniciativa ciudadana, como una herramienta para detener la violación de sus derechos fundamentales ante la pasividad del Estado.

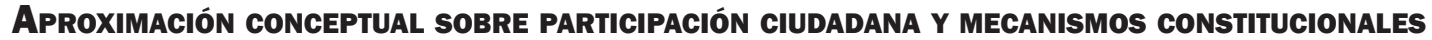

Resulta necesario, iniciar con un acercamiento conceptual que permita comprender los posibles alcances de la participación ciudadana en la generación de control político y la creación de políticas públicas que respondan a las necesidades insatisfechas de la población, por lo cual es pertinente antes, el análisis también de cómo puede generarse, una apropiación de conocimientos que efectivamente puedan ser puestos en práctica por la ciudadanía.

Por ello, se debe entender el concepto de control político el cual reside por mandato constitucional en el Congreso de la República y se ejerce ante el gobierno nacional y las instituciones del poder ejecutivo y en virtud del Acto legislativo 02 de 2007 se amplía el espectro del control político, en el que se incluyen a las asambleas departamentales 
y a los consejos municipales, como lo dice el profesor Henao Hidrón en el sentido de expresar que el Congreso de la República cuenta con una serie de acciones mediante las cuales solicita explicaciones a las autoridades del poder ejecutivo sobre actuaciones $u$ omisiones frente a mandatos legales que están bajo su responsabilidad, o por denuncias que la ciudadanía presente ante el Congreso, pudiendo estos ser objeto de sanciones por parte del Congreso bajo un procedimiento previo, en procura de garantizar y remover el equilibrio de poderes en el Estado. (Henao Hidron, 2004).

En el mismo sentido lo expresa Paolo Biscaretti di Ruffia cuando indica:

El control político sobre el gobierno lo realizan las cámaras con base en dos frentes: la actividad político-administrativa y la actividad financiera. Respecto de la primera, señala que el control se logra por medio de preguntas, que consisten en solicitar aclaraciones formuladas por escrito al Gobierno para saber si un determinado hecho es cierto o si una información es exacta, donde se puede solicitar una respuesta escrita u oral; interpelaciones, que se refieren específicamente a los motivos o fines de la conducta del Gobierno respecto de algún aspecto de su política -se trata de un verdadero debate-; mociones, que se presentan para "obtener una discusión y la consiguiente votación de un preciso 'orden del día' sobre un objeto determinado, destinado a comprometer, respecto del mismo, la actividad posterior del Gobierno", y en las cuales se pueden proferir resoluciones en las que contengan juicios respecto de lo actuado por el gobierno. (Paolo Biscaretti di Ruffia 1987, como se cito en Rincon, 2013, pág. 361)

Lo anterior es imperante teniendo en cuenta que el Estado Social de Derecho reposa en el control que realizan cada uno de los poderes frente a los otros y en el equilibrio de poderes siendo precisamente el control político, uno de los instrumentos para exigir al ejecutivo el cumplimiento en el desarrollo y ejecución de las normas y funciones en los distintos ámbitos en los que se legisla, según las necesidades de cada momento.

Otro de los conceptos que resulta de gran relevancia para evidenciar la importancia de la participación ciudadana, tiene que ver con las políticas públicas, desarrolladas por el gobierno, y que teóricamente el profesor Manuel Quinche ha definido:

Un conjunto de actividades, decisiones o medidas diseñadas por el Estado, y en ocasiones también llevadas a cabo directamente por él, mediante las cuales se pretende intervenir en determinadas situaciones previamente identificadas -que por lo general corresponden a situaciones socialmente percibidas como problemáticas- con el propósito de cumplir determinados objetivos -que usualmente corresponden a la intención de mitigar o de corregir esas situaciones problemáticas. (Quinche \& Rivera, 2010, pág. 118).

En el mismo sentido ha expresado que en dichas políticas públicas han de coexistir 4 elementos que permiten evidenciar la existencia de una política pública; en primer lugar, deberá existir una circunstancia específica que deba ser atendida, bien sea por considerarse como de urgencia o porque confluyen intereses políticos que posibilitan la intervención de la situación específica. También, se deberá demarcar el propósito y la finalidad de la intervención que se pretende realizar frente a la circunstancia que 
se ha seccionado. Posteriormente se deberán planear, desarrollar y ejecutar una serie de actividades o medidas con el fin de alcanzar el propósito de dicha intervención, y finalmente se debe evidenciar la participación directa o indirecta del gobierno como representación del Estado (Quinche \& Rivera, 2010) en las distintas fases de la política pública, siendo posible dividirlas en tres momentos que no necesariamente deban ejecutarse uno tras otro, si no que dependiendo de la situación, podrán confluir en un mismo momento como, seguidamente lo expresa el profesor Quinche al indicar:

Las políticas públicas están compuestas de varias etapas, que comprenden: 1) la formulación, luego de un proceso de estudio y discusión, de una serie de medidas por medio de las cuales se pretende solucionar determinado problema; 2) la implementación concreta de las medidas formuladas, y 3) la evaluación o seguimiento de las medidas adoptadas y de los efectos que ha producido su implementación. Esta diferenciación entre las distintas etapas que conforman una política pública tiene la ventaja de desagregarlas en segmentos que facilitan su análisis, permitiendo así una percepción más clara del alcance del control judicial que se ejerce sobre ellas (Quinche \& Rivera, 2010, pág. 118).

Ahora bien, en materia jurisprudencial también es posible identificar algunas condiciones sine quan non para la existencia de una verdadera política pública que pretenda garantizar el goce efectivo de un derecho fundamental, como lo expresa la Corte Constitucional en la Sentencia T-760 de 2008, en la que dijo:

Concretamente, la jurisprudencia constitucional ha precisado tres condiciones básicas, a la luz de la Constitución Política, que debe observar toda política pública orientada a garantizar un derecho constitucional. (...)

3.3.11. La primera condición es que la política efectivamente exista. No se puede tratar de unas ideas o conjeturas respecto a qué hacer, sino un programa de acción estructurado que le permita a la autoridad responsable adoptar las medidas adecuadas y necesarias a que haya lugar.

3.3.12. La segunda condición es que la finalidad de la política pública debe tener como prioridad garantizar el goce efectivo del derecho. En tal sentido, por ejemplo, no puede tratarse de una política pública tan sólo simbólica, que no esté acompañada de acciones reales y concretas.

3.3.13. La tercera condición es que los procesos de decisión, elaboración, implementación y evaluación de la política pública permitan la participación democrática. En tal sentido, la jurisprudencia ha considerado inaceptable constitucionalmente que exista un plan (i) 'que no abra espacios de participación para las diferentes etapas del plan', o (ii) ‘que sí brinde espacios, pero éstos sean inocuos y sólo prevean una participación intrascendente. (subrayas agregadas) (Corte Constitucional, 2008 Sala Segunda de Revision, numeral 3.3. y ss.)

3.3. Resulta de lo anterior evidente que deben coexistir distintos elementos para la correcta ejecución de una política pública, entre ellos, como lo expresó la Corte Constitucional, la participación democrática que no es otra cosa sino brindar la oportunidad real y efectiva a la población a la que deberá dirigirse la política pública, de intervenir de forma directa 
en las distintas etapas de la política pública, así como permitir la intervención de todos los sectores que tengan algún interés o implicación en la aplicación de ella, pero ante todo evitando que este último elemento sea meramente simbólico.

En tal sentido, resulta también relevante brindar una aproximación a lo que significa e implica la ciudadanía entendida como el conjunto de derechos y deberes de los ciudadanos que hacen parte de un Estado y que, por ende, se les atribuye el derecho de involucrarse en la toma de decisiones, e implica la garantía en cabeza del Estado frente a la protección de los derechos y libertades (Favinha y Navarro Medina, 2012 pág. 3); además de ello, resalta Bolívar (2007) que la ciudadanía debe entenderse: "Cómo la práctica de una actividad moralmente deseable para una revitalización de la democracia. Esto implica educar en un conjunto de competencias cívicas, en tanto que ciudadano, con una metodología de debate y deliberación sobre los asuntos comunes" . Bolívar, A. 2005, como se cito en (Favinha y Navarro Medina, 2012 pág. 3).

Ahora bien, en correlación con los aspectos que componen la ciudadanía, se puede constatar que la Carta Política, ha dotado a la ciudadanía de una serie de mecanismos de participación, con los que se pretende dar contenido a los principios que rige el Estado Social de Derecho como lo es la pluralidad, la democracia y la participación, por lo cual, se han dividido en tres grandes grupos, sin que sea una asignación estricta, si no ilustrativa; es así que existen mecanismos constitucionales de tipo: judicial, político y administrativo, según sea su naturaleza, procedimiento y las exigencias o solemnidades de cada una, los cuales procuran el cumplimiento de objetivos en principio distintos, sin embargo, guardan relación en el sentido de la participación de la ciudadanía en cada uno de los mecanismos que a continuación se enuncian:

De forma general, es posible asignar a los mecanismos judiciales: la Acción de Tutela, Acción Popular, Acción de Grupo y Acciones de Constitucionalidad (Inconstitucionalidad, Inexequibilidad y Nulidad por Inconstitucionalidad), que tienen como característica un procedimiento especial, ágil, preferente y sumario, pudiendo ser interpuestas directamente por el ciudadano (Moreno \& Angel, 2018, pág. 36) .

Por otra parte, se identifican mecanismos de naturaleza política, que están directamente relacionados con la posibilidad de la población de hacer un control de las actuaciones de la administración desde la comunidad, en el entendido que algunas de las acciones que se presentan a continuación, requieren de la participación de una pluralidad de ciudadanos para lograr su efectividad, es el caso de: la Revocatoria de Mandato, veedurías ciudadanas, cabildo abierto, referendo, plebiscito y consulta popular (Moreno y Ángel, 2018).

Finalmente, se pueden identificar mecanismos de tipo administrativo, con los que se pretende una interacción directa con la administración en procura de obtener una 
respuesta de forma pronta por parte de ella, respecto a situaciones concretas que puedan requerir los ciudadanos; además, no son mecanismos exclusivos para hacerse valer ante la administración, sino que pueden ser ejercidos frente a particulares, según lo dispuesto tanto en la Constitución Política, como en la Ley 1755 de 2015, por medio de la cual se regula el Derecho Fundamental de Petición.

En términos generales, son estos los mecanismos o acciones a las que tienen derecho los ciudadanos en Colombia; sin embargo, ello no quiere decir que la simple existencia de los mecanismos aquí expuestos, implica su efectiva aplicación, cumplimiento o eficiencia, pues para ello es importante también contar con una serie de competencias ciudadanas que permitan hacer uso y disponer en debida forma de dichos mecanismos; en ese sentido, se hará una aproximación frente a lo que se ha desarrollado sobre competencias en relación con la ciudadanía.

En ese sentido, el primer concepto que se tiene en cuenta proviene del Ministerio de Educación Nacional de Colombia (MEN), que determina la competencia como un "saber hacer", como el poner el conocimiento a disposición de la realización de acciones o productos que permitan enfrentar los problemas de la vida cotidiana (Ley 1994). Esta concepción va ligada a la educación y formación de las personas en aras de lograr distintas capacidades o habilidades en el "saber hacer"; sin embargo, dichas capacidades no siempre son desarrolladas en debida forma debido a una multiplicidad de factores que así lo determinan; por ejemplo, el acceso limitado a la educación formal, tanto en la educación pública como privada y el contexto social actual, en el que existe una apatía por intervenir en la toma de decisiones que le competen a la ciudadanía (Murillo Castaño \& Castañeda , 2007, pág. 5).

Otros doctrinantes se han referido a las competencias desde el aspecto del lenguaje expresando al respecto que las competencias se refieren a un proceso de aprendizaje en un contexto y uso determinado, que desembocarán en la apropiación de conocimientos que sirven de herramienta o formas de expresarse por parte de los ciudadanos (Ruiz Silva \& Chaux Torres, 2005).

También, es posible identificar tres tipos de competencias, teniendo en cuenta que el componente de la ciudadanía implica una interrelación entre las personas, lo que genera la necesidad de que la competencia que se llegare a adoptar o apropiar, está directamente relacionada con la posibilidad de ponerla en práctica, no solo en intereses personales si no en el bienestar de un grupo o comunidad (Ruiz Silva \& Chaux Torres, 2005, pág. 32); en ese sentido, la primera competencia y de relevancia para esta investigación es descrita por Silva y Chaux como una competencia cognitiva:

Las competencias cognitivas son las capacidades para realizar diversos procesos mentales. En este caso, capacidades para llevar a cabo procesos que favorecen el ejercicio de la ciudadanía. 
Una de éstas es la habilidad para tomar diversas perspectivas o, en otras palabras, para ponerse mentalmente en la posición del otro. Esta competencia favorece tanto la convivencia pacífica como la participación democrática y la pluralidad y valoración de las diferencias. Lograr acuerdos de beneficio mutuo e interactuar en forma colaborativa con otras personas (Ruiz Silva \& Chaux Torres, 2005, pág. 33).

Es necesario en la lectura de esta definición, traer a colación la definición que se dio frente a la ciudadanía en tanto se debe contar con competencias cívicas y la participación activa en las decisiones que afecten a la población. Además de ello, es posible hacer la analogía con la adquisición de conocimientos que permitan para sí mismo o para alguien más, brindar las herramientas que faciliten la protección de los derechos de los ciudadanos a través del uso de los mecanismos de protección de derechos.

La siguiente competencia es descrita como una competencia comunicativa, que implica un entendimiento y adaptación de las personas a un "entorno social", aquel ámbito en el que se desenvuelve y en el cual se pretende aplique, además su expresión por medio del lenguaje le permitirá entablar relaciones sociales y personales que creen lazos comunitarios. Finalmente, está la competencia emocional, que se refiere a la capacidad del individuo para: "Identificar las emociones propias y las de los otros, y responder a ellas de forma constructiva." (Ruiz Silva \& Chaux Torres, 2005, pág. 42). Esta definición también incluye un elemento que los autores Silva y Chaux han concebido como empatía en referencia a la capacidad de comprender la situación de la otra persona y procurar ayuda desde el despliegue de estas competencias

Ahora bien, en cuanto al componente de las competencias desde el ámbito de la ciudadanía, es acertado decir que la educación formal que se brinda por parte del Estado y desde las instituciones de educación, puede resultar insuficiente teniendo en cuenta el limitado acceso a dichos servicios, entre otros, y es en ese escenario que las competencias ciudadanas resultan de gran relevancia, si de lo que se trata es de dotar a la ciudadanía de las herramientas que les brinden un "saber hacer" específicamente en lo que se refiere a la protección de sus derechos, traducido en la apropiación de conocimientos encaminados a lograr una efectiva protección de sus garantías, a través del uso de los mecanismos constitucionales que prevé la Constitución Política.

Conforme con lo anterior, resulta de vital importancia la no dependencia exclusiva de la educación que proviene del Estado como una de sus obligaciones y principios, si no que se deben incentivar alternativas de formación y educación que facilite ampliar el espectro y el impacto de la formación en la ciudadanía en procura de formar y lograr la apropiación de competencias que les permitan específicamente, en el enfoque de este trabajo, la apropiación y empoderamiento de los distintos mecanismos constitucionales de protección de derechos que existen puntualizando en uno de los mecanismos de tipo judicial como lo es la Acción de tutela en consideración a que por su naturaleza 
está llamado a la defensa de derechos fundamentales y sin embargo; se demostrara como también resulta aplicable para la defensa de derechos colectivos y permite la intervención de un organismo independiente del Gobierno Nacional o los Gobiernos Territoriales como lo es la Corte Constitucional en procura del cumplimiento de las obligaciones de los primeros.

Es así que los conceptos descritos en la presente sección resultan de vital importancia para en términos generales verificar que incentivar en la ciudadanía, la participación política activa, en definitiva, impacta la vida personal y social de una comunidad que cuente con los conocimientos y las herramientas apropiadas para proteger tanto derechos individuales como colectivos.

Es posible entonces afirmar, que existen mecanismos los cuales han sido creados específicamente para ejercer control político y control a políticas públicas frente a las actuaciones administrativas como lo son los mecanismos denominados: "mecanismos políticos" (Moreno y Ángel, 2018. Pág. 37), sin embargo, resulta interesante exponer en adelante, cómo es que a través de un mecanismo constitucional que en principio pretende la protección de derechos individuales como lo es la acción de tutela, de forma indirecta también ha servido para que se ejerza control político y se generen pautas para la creación de políticas públicas, claro ejemplo de ello se podrá evidenciar en el desarrollo de la sección que a continuación se presenta, teniendo como referencia la perspectiva que surge en materia de salud en Colombia, con la Sentencia T-760 de 2008 expedida por la Corte Constitucional, como antecedente histórico en esta materia específica, en la que la acción de la ciudadanía resulta fundamental en la generación y progresión en materia de políticas públicas y control político a las actuaciones $u$ omisiones del Estado en materia de salud.

\section{Contexto histórico legal de la salud en Colombia y control político ejercido por la Corte Constitucional en la Sentencia T-760 de 2008}

Para el desarrollo de esta sección se pretende ejemplificar cómo desde el ejercicio de un mecanismo de protección de derechos fundamentales, es posible además, lograr un control político y control en la creación de políticas, por tal motivo, se trae a colación en primer lugar, el proceso histórico en que surge la sentencia T-760 del 2008 proferida por la Corte Constitucional desde el enfoque de la salud, considerando que el momento coyuntural en que se desarrolla la sentencia, implicaba la necesidad de la actuación inmediata por parte de las autoridades tanto nacionales como territoriales en vista de la omisión legislativa y administrativa que se vivió en ese momento, lo cual se consiguió en virtud de una serie de ordenes emanadas de un cuerpo colegiado independiente de cualquier autoridad estatal y quien tiene la facultad para exigir a las entidades públicas y privadas el cumplimiento de las obligaciones que tienen a cargo 
en tratándose de la protección y garantía de los derechos fundamentales. En segunda medida, se presenta la identificación del control político y los parámetros mínimos que deben contener las políticas públicas en cuanto al contenido prestacional en lo que a derechos fundamentales se refiere, producto de las ordenes emanadas de la decisión de la sentencia, como resultado de una acción ejercida por la ciudadanía.

\section{Contexto histórico legal de la salud en Colombia}

Conforme con lo anterior, el punto de partida es la contextualización de lo que ha sido la evolución histórico legal de la salud en Colombia, bajo un marco normativo que ha sufrido diferentes cambios hasta el año 2008, mismo año en el que se profirió la sentencia T-760 bajo la cual se logró evidenciar el control político ejercido por la Corte Constitucional, con una serie de disposiciones que llevaron a la creación de políticas públicas por parte del gobierno, para dar cumplimiento a lo ordenado en la parte resolutiva de dicho fallo.

El primer cuerpo normativo en materia de salud en Colombia data de 1886 cuando se expide la Ley 30 que crea las "Juntas de Higiene en la Capital de la República y en los Departamentos y Ciudades principales”; esta ley creó precisamente órganos que se encargarían de la salubridad pública. Esta misma norma también expresa el monto económico que se debe aplicar para el sostenimiento de las juntas de higiene, puesto que por primera vez el Estado intenta crear instituciones que se encargarían de temas relacionados con la salud. Bajo estos postulados, los temas de salud a los que se refería dicha ley eran generalmente sobre exposiciones ambientales, epidémicos, entre otros (Ley 30, 1886).

Posteriormente, se expiden por parte del Congreso de la Republica diferentes leyes que intentan de alguna manera generar cambios en la salubridad, pero mayormente, referidos a cambios institucionales que a cambios netamente sociales; algunas de estas son:

i) Ley 33 de 1913 "Por la cual se organiza la higiene nacional pública y privada”, donde principalmente crea comisiones sanitarias, junto a las ya creadas juntas de higiene.

ii) Ley 32 de 1918 "Sobre organización y dirección de los Lazaretos de la República y reorganización de la Dirección Nacional de Higiene" esta ley principalmente creo centros hospitalarios para tratar la lepra.

iii) Ley 96 de 1938, "Por la cual se crean los Ministerios de Trabajo, Higiene y Previsión Social y de la Economía Nacional". Esta norma precisa más importancia debido a que bajo esta ley se eliminan las juntas de higiene, y se crea un ministerio que se encargara de la salud en Colombia. 
Hasta el año 1950 el modelo sanitario en el país se demonio modelo "higienista" y fue el primer periodo que tuvo Colombia sobre salud pública. El modelo higienista es descrito de manera asertiva por José Jaime Castaño Castrillón donde dice: "por la cual la intervención del Estado se limitaba a atender aspectos de carácter sanitario, y la intervención básica en salud debía ser financiado por los usuarios y algunas entidades de caridad" (Castrillón, 2013). Otro aspecto importante en 1950 es la creación de dos entidades: la Caja Nacional de Previsión "encargada de la salud de los empleados públicos" y el Instituto Colombiano de los Seguros Sociales "que hacía lo propio para los empleados del sector privado" (Castrillón, 2013).

El segundo periodo de la salud en Colombia es el comprendido entre 1970 hasta 1989, dentro de los temas importantes, expresa José Castrillón: "Se crea el Sistema Nacional de Salud, en el cual los recursos estatales para la salud eran transferidos directamente a la red de hospitales públicos" (Castrillon, 2013). Dentro de este segundo periodo de la salud en Colombia se expide la ley 9 de 1979 "por la cual se dictan medidas sanitarias", ley que resulta de gran importancia principalmente porque empieza hablar de la preservación, restauración y mejoramiento de las condiciones sanitarias, de los residuos sanitarios y del cuidado con el medio ambiente.

El tercer periodo que vive Colombia, es un periodo más amplio pese a que no se tiene claridad de la fecha de su terminación, debido a que algunos historiadores lo describen hasta la actualidad incluyendo reformas e intervenciones a diferentes entidades estatales y otros, como José Castrillón, lo delimitan hasta el año de 1993. Sin embargo, se tiene claridad que en 1990 se expide la Ley 10: "Por la cual se reorganiza el Sistema Nacional de Salud y se dictan otras disposiciones". El cambio principal que presentó esta norma con relación al "modelo higienista" es que habla de la salud como un servicio íntegro y gratuito en los servicios básicos para todos los habitantes del país, compuesto por entidades descentralizadas y entidades privadas autorizadas por el Estado; sin embargo, esta ley duró apenas hasta 1993, en razón a todo el proceso que vivió Colombia en términos sociales, económicos y políticos, como resultado de la expedición de la Constitución Política de 1991 y la posterior expedición de la Ley 100 de 1993 "por la cual se crea el sistema de seguridad social integral y se dictan otras disposiciones", ley que actualmente rige en Colombia.

La Ley 100 de 1993 es de gran importancia para este artículo de investigación debido a que es precisamente sobre esta, que la Corte Constitucional en la Sentencia T-760 de 2008 interviene para ejercer un control político y genera lineamientos mínimos de una política pública frente a los derechos fundamentales, para el presente estudio, el derecho a la salud. Dicha norma se caracteriza por generar especialidad en diferentes temas como: pensión, salud, y riesgos profesionales. Asimismo, creó cuatro sistemas importantes para el país, que son: el sistema general de pensiones, el sistema general de seguridad social en salud, el sistema general de riesgos profesionales y los servicios sociales complementarios. 
La ley en mención, suponía un cambio en Colombia debido a que pretendía la cobertura de seguridad social integral para toda la población, sin discriminación alguna, tema que fue problemático con leyes anteriores.

Sin embargo, a pesar de ser innovadora en su contenido, por diferentes motivos su aplicación no se dio de manera inmediata y su pedagogía con las entidades prestadoras de servicio no fue la mejor, puesto que, después de entrar en vigencia, las entidades prestadoras de servicio en salud no tenían claridad de la aplicación de la norma respecto del contenido de los servicios de salud.

Por lo anterior, la Sentencia T-760 de 2008 implicó, para el sector salud en Colombia, un cambio estructural y de las entidades, en procura de que se produjera una aplicación real y efectiva de la Ley 100 de 1993, entre otros importantes cambios sociales, que durante todo este último periodo estaba en mora de ejecutarse tanto por parte del legislador, como por las autoridades en materia de salud del orden nacional y territorial.

\section{Control político de la Sentencia T-760 de 2008}

Con la expedición de la sentencia T-760 de 2008, la Corte Constitucional genera diferentes cambios en la estructura del Sistema General de Seguridad Social en Salud, que se tratarán a fondo en la presente sección, logrando evidenciar cómo a través de la acción constitucional- acción de tutela ejercida por ciudadanos que padecían de forma directa la inaplicación en debida forma de la Ley 100 de 1993 debiendo recurrir a este mecanismo para que sus derechos fueran reconocidos y, en consecuencia generando control político a las entidades públicas y privadas encargadas de la garantía del derecho a la salud.

También, la Corte Constitucional estableció los parámetros o lineamientos de obligatorio cumplimiento por parte del gobierno para generar políticas públicas en salud, en los que se incluyen órdenes directas que permitan el cumplimiento de sus órdenes. Siendo esto en esencia, el análisis que a continuación se desarrolla:

El primer punto al que se refiere la sentencia T-760 de 2008 es al problema jurídico. Es de esta manera que la Corte Constitucional dentro de la sentencia dice:

La Corte Constitucional aborda varios casos en los que se invoca la protección del derecho a la salud -concretamente, el acceso a servicios de salud que se requieren-, cuya solución ha sido clara y reiterada en la jurisprudencia de esta Corporación. Estos casos se refieren a diversas situaciones en las cuales el acceso a los servicios de salud requerido fue negado" (Corte Constitucional, 2008 Sala Segunda de Revision, numeral 2.)

En este primer punto, la Corte Constitucional expresa que el problema central para que los ciudadanos se acercaran pretendiendo la protección constitucional, tiene que ver 
con la vulneración continuada del derecho a la salud. En este sentido los temas más concurrentes que evidenció la Corte Constitucional y sobre los cuales profirió su decisión fueron: i) acceso a servicios de salud contemplados en el plan obligatorio de salud, POS, sometidos a pagos moderadores; ii) acceso a servicios de salud no incluidos dentro del POS; iii) acceso a los servicios de salud que requiere un menor para su adecuado desarrollo; iv) reconocimiento de incapacidades laborales cuando no se cumplen los requisitos de pago oportuno; v) acceso a los servicios de salud en condiciones de integralidad; vi) acceso a los servicios de salud de alto costo y para tratar enfermedades catastróficas, así como a los exámenes diagnósticos; vii) acceso a los servicios de salud requeridos por personas vinculadas al Sistema de Salud (Sentencia T-760, 2008).

Posteriormente, la Corte Constitucional realizo un análisis de cada uno de los casos presentados por los ciudadanos respecto de la posible vulneración de sus derechos; teniendo como eje fundamental los 22 casos presentados, la negación del acceso a la salud, así como el desconocimiento por parte de las entidades prestadoras del servicio en salud, respecto de los servicios que se encontraban dentro del Plan Obligatorio de Salud (en adelante POS), teniendo en cuenta que existían algunos servicios que no se encontraban dentro de la cobertura, situación que actualmente ha cambiado, puesto que las EPS (Entidad Promotora en Salud) se rigen a Resoluciones anuales del Ministerio de Salud, en el cual se establecen con exactitud el contenido del Plan de Beneficios en salud y cuáles son los servicios que se encuentran excluidos del mismo.

Debido a todas estas inconformidades presentadas por los ciudadanos a través de la acción de tutela, la Corte Constitucional decide amparar en su mayoría el derecho a que tuvieran los ciudadanos ${ }^{1}$, pero consecutivamente y lo más trascendental es el control político que ejerce frente a las instituciones del Estado específicamente al Ministerio de la Protección Social, a la Comisión de Regulación en Salud y al Consejo Nacional de Seguridad Social en Salud. Una de las órdenes más importantes frente al control político dada por parte de la Corte Constitucional dentro de la sentencia T-760 de 2008 tiene que ver con los planes de beneficios:

Décimo sexto.- Ordenar al Ministerio de la Protección Social, a la Comisión de Regulación en Salud y al Consejo Nacional de Seguridad Social en Salud, adoptar las medidas necesarias, de acuerdo con sus competencias, para superar las fallas de regulación en los planes de beneficios asegurando que sus contenidos (i) sean precisados de manera clara, (ii) sean actualizados integralmente, (iii) sean unificados para los regímenes contributivo y subsidiado y, (iv) sean oportuna y efectivamente suministrados por las Entidades Promotoras de Salud.

Esta regulación también deberá (i) incentivar que las EPS y las entidades territoriales garanticen a las personas el acceso a los servicios de salud a los cuales tienen derecho; y (ii) desincentivar la denegación de los servicios de salud por parte de las EPS y de las entidades territoriales.

1. La Corte Constitucional resuelve la Sentencia T 760 DE 2008 con fundamento en los expedientes -1281247, T-1289660, T-1308199, T-1310408, T-1315769, T-1320406, T-1328235, T-1335279, T-1337845, T-1338650, T-1350500, T-1645295, T-1646086, T-1855547, T-1858995, T-1858999, T-1859088, T-1862038, T-1862046, T-1866944,

T-1867317, y T-1867326, los cuales fueron objeto de protección individual por parte de la Corte Constitucional de forma paralela y consecuente con la Expedición de la Sentencia T 760 DE 2008. 
Para dar cumplimiento a esta orden, se adoptarán por lo menos las medidas relacionadas en los numerales décimo séptimo a vigésimo tercero (T-760, 2008).

En las siguientes órdenes que también generan control político a las entidades del Sistema general de seguridad social se trataron los siguientes temas:

Actualización anual del POS, participación de la comunidad médica, los usuarios del sistema de salud también deben participar dentro de esta actualización, explicar los temas que están excluidos y los que no se prestaran dentro del POS, debe darse actualización a los planes una vez al año por lo menos, deben realizarse informes que deben ser enviados a la comisión nacional en salud, identificar las entidades promotoras de servicio en salud que con mayor medida niegan el servicio de salud, unificar planes de servicio para los niños y las niñas (T-760, 2008).

Es a través de las ordenes expuestas en los párrafos anteriores, que el Gobierno Nacional debió a partir de la expedición de la sentencia en cuestión, anualmente expedir una resolución en la que se actualice el POS, específicamente en cuanto al plan de beneficios en salud se refiere.

Además de generar control político, la Corte Constitucional al comprobar la inaplicación de políticas públicas en salud ya existentes, interviene con el fin de que las órdenes impartidas para la restructuración del sistema general de seguridad social en salud, estén sustentadas bajo una política pública que cumpla con parámetros mínimos, permitiendo su aplicación efectiva. De esta manera es como se establecen los lineamientos que el Gobierno Nacional, deberá seguir en la creación de una política en salud, que en definitiva conlleve a la correcta ejecución del nuevo modelo de Seguridad Social en Salud.

La Corte Constitucional basa como eje fundamental de la política pública en salud los derechos humanos, pues expresa "esta decisión se adoptó considerando la estrecha relación entre la salud y el concepto de la dignidad humana, elemento fundante del Estado Social de Derecho que impone a las autoridades y a los particulares el trato a la persona conforme con su humana condición" (Corte Constitucional, 2008). También, la Corporación ha ordenado que cualquier política pública que se pretenda desarrollar en materia de salud, debe ser siempre orientada con un componente de Derechos Humanos, con el fin que el derecho fundamental a la salud sea solidario, universal y de efectivo acceso.

En este orden de ideas, ordena la Corte que el Estado al identificarse como Estado Social de Derecho debe encaminar los recursos económicos necesarios para dar cumplimiento al acceso a la salud, motivo por el cual señala: "que el derecho a la salud es, autónomamente, un derecho fundamental y que, en esa medida, la garantía de protección debe partir de las políticas estatales, de conformidad con la disponibilidad de los recursos destinados a su cobertura (Corte Constitucional, 2008), es de esta manera, que se podrá dar un verdadero cumplimiento de las políticas públicas ordenadas al Gobierno nacional referente al cumplimiento del derecho a la salud. 
Otro tipo de elementos que deben hacer parte de la política pública en salud son:

i. Las personas deben hacer parte de la creación de las políticas públicas, situación que es asertiva por parte de la Corte, puesto que precisamente esta política estatal va dirigida a la sociedad.

ii. Adoptar decisiones con una correcta planificación y aplicación, y las estrategias que se necesiten para el fortalecimiento del derecho a la salud.

iii. Se debe tener en cuanta al momento de la creación de política pública, la comunidad médica para saber que estará incluido o excluido del plan obligatorio de Salud.

De esta manera, la Corte Constitucional plantea dos tipos de obligaciones para la creación de las políticas estatales. Las primeras, denominadas "obligaciones básicas" ${ }^{2}$ las cuales hacen parte del núcleo esencial del derecho, con lo cual, genera que el cumplimiento de dichas obligaciones no puedan ser diferidas en el tiempo por parte del Estado, entre otras; es posible identificar algunas de esas obligaciones desde la perspectiva legislativa y como es que el Estado, desde la producción normativa, debe cumplir con la obligación de proteger, y respetar el goce de los derechos fundamentales, a través de decisiones institucionales que establezcan los parámetros claros frente al contenido y alcance que se pretende dar a los derechos. (Corte Constitucional, 2012).

Las segundas, llamadas "obligaciones prioritarias" 3 que hacen referencia a aquellas actividades que debe desplegar el Estado y las entidades respectivas, para propiciar el desarrollo y mejora constante del contenido prestacional de los derechos fundamentales, que no es diferente a una serie de facetas que puede tener el derecho, y es así como surge la obligación del Estado en cumplir con el principio de progresión y no regresividad, (Corte Constitucional, 2012)el cual afirma que el Estado en la medida de su capacidad económica y presupuestal, deberá avanzar en la cobertura y eficacia de dicha faceta de los derechos. Ello no implica de ninguna forma, que el Estado pueda a voluntad abstenerse de la implementación de todas las acciones tendientes a mejorar ese contenido prestacional, o que realice acciones que vallan en contravía de los avances que se han logrado en dicha materia, debiendo tener presente y a manera de ejemplo la existencia de preceptos internacionales como lo es Comité de Derechos Económicos,

2 "a) Velar por la atención de la salud genésica, materna (prenatal y postnatal) e infantil; b) Proporcionar inmunización contra las principales enfermedades infecciosas que tienen lugar en la comunidad; c) Adoptar medidas para prevenir, tratar y combatir las enfermedades epidémicas y endémicas; d) Impartir educación y proporcionar acceso a la información relativa a los principales problemas de salud en la comunidad, con inclusión de los métodos para prevenir y combatir esas enfermedades; e) Proporcionar capacitación adecuada al personal del sector de la salud, incluida la educación en materia de salud y derechos humanos." (Comité de Derechos Económicos, Sociales y Culturales. Observación General N¹4 (2000).

3“a) Garantizar el derecho de acceso a los centros, bienes y servicios de salud sobre una base no discriminatoria, en especial por lo que respecta a los grupos vulnerables o marginados; b) Asegurar el acceso a una alimentación esencial mínima que sea nutritiva, adecuada y segura y garantice que nadie padezca hambre; c) Garantizar el acceso a un hogar, una vivienda y unas condiciones sanitarias básicos, así como a un suministro adecuado de agua limpia potable; d) Facilitar medicamentos esenciales, según las definiciones periódicas que figuran en el Programa de Acción sobre Medicamentos Esenciales de la OMS; e) Velar por una distribución equitativa de todas las instalaciones, bienes y servicios de salud; f) Adoptar y aplicar, sobre la base de las pruebas epidemiológicas, una estrategia y un plan de acción nacionales de salud pública para hacer frente a las preocupaciones en materia de salud de toda la población; la estrategia y el plan de acción deberán ser elaborados, y periódicamente revisados, sobre la base de un proceso participativo y transparente; esa estrategia y ese plan deberán prever métodos, como el derecho a indicadores y bases de referencia de la salud que permitan vigilar estrechamente los progresos realizados; el proceso mediante el cual se concibe la estrategia y el plan de acción, así como el contenido de ambos, deberá prestar especial atención a todos los grupos vulnerables o marginados." Observación General N¹4 (2000). 
Sociales y Culturales de la ONU (Comité DESC), el cual en su Observación General No. 3, enuncia la naturaleza de las obligaciones contraídas por los Estados que suscribieron el Pacto. ${ }^{4}$ (Corte Constitucional, 2012).

En concordancia con lo anterior, la Corte Constitucional ha indicado que existe una clasificación de obligaciones que devienen de los derechos fundamentales, que en ese sentido, implican para el Estado una serie de acciones "positivas" y "negativas" encaminadas a lograr la efectividad de los derechos fundamentales. Es así que en la sentencia T-760 de 2008 se resalta la importancia y trascendencia de que el Estado en cumplimiento de sus obligaciones, despliegue todas las acciones ("positivas") necesarias para la garantía y protección de un derecho fundamental. Adicionalmente pone de presente la importancia de que en momentos determinados también deberá abstenerse (“negativas) de ejecutar alguna acción por sí mismo o por intermedio de entidades privadas, que ejecuten acciones en contravía de la protección y garantía de los derechos fundamentales de las personas, lo que podría eventualmente traducirse en: "Dejar sin efecto una decisión administrativa o una determinada regulación." (Corte Constitucional, 2008).

Lo anterior da cuenta del alcance de la intervención de la Corte Constitucional a partir del ejercicio de la ciudadanía de los mecanismos constitucionales de protección de derechos, específicamente la acción de tutela, en virtud de la evidente problemática que en materia de salud existió en Colombia, sin embargo, es importante entender que dichos parámetros anunciados, tienen una aplicación transversal en cuanto a la producción y creación de políticas públicas en que se vean inmersos derechos fundamentales de las personas, con lo cual, resulta de gran relevancia entender que este tipo de fallos aportan no solo a la solución de conflictos particulares, puesto que se convierten en hojas de ruta o guías que deben cumplir las entidades nacionales o territoriales al momento de intervenir derechos fundamentales mediante políticas públicas.

El papel de la educación en el ejercicio del control político. Experiencia del Diplomado de Competencias Ciudadanas y Mecanismos Constitucionales en UNICOC

En esta sección resulta importante hacer algunas apreciaciones en lo que tiene que ver con la pedagogía y la educación ciudadana, de forma específica en mecanismos constitucionales de protección de derechos, como los medios sobre los cuales se pretende que la ciudadanía logre la apropiación de conocimiento que le permitan acceder de forma efectiva a la protección de sus derechos, del mismo modo, generar el control político a través de su participación activa.

4EI Comité citado es el órgano de la ONU encargado de controlar la aplicación del Pacto y, por lo tanto, el intérprete autorizado del Instrumento. Si bien sus observaciones no hacen parte del bloque de constitucionalidad, en el sentido de ingresar directamente al orden jurídico colombiano como normas vinculantes, su observación es imprescindible para que el Estado colombiano cumpla de buena fe sus obligaciones en materia de derechos humanos. Como criterio de interpretación, la Corte siempre que lo considera pertinente acude a la interpretación del Comité DESC pues, salvo en los aspectos en que el orden interno prevea mayores garantías que las establecidas en el Pacto, puede considerarse que su interpretación busca dar el máximo de efectividad normativa a los derechos humanos contenidos en el PIDESC. 
En ese sentido es válido afirmar que en definitiva la educación de los ciudadanos cobra importancia en procura de formar e incentivar las competencias cívicas que les permitan por sus medios, participar activamente de las decisiones que se tomen en el Estado, que puedan lograr la efectiva protección de derechos a través de los mecanismos constitucionales de protección de derechos que, en el caso colombiano, la Constitución Política prevé. Así las cosas, frente a la educación de la ciudadanía, Audigier (1998) señala que a través del Consejo de Europa se ha trabajado para que sean adoptadas en la elaboración de políticas, legislaciones y prácticas de los Estados. Expresando:

La Educación para la Ciudadanía democrática (EDC) es un concepto multifacético, relativo a aspectos políticos, sociales, económicos, culturales, ambientales y éticos de las sociedades democráticas modernas. Un proceso de aprendizaje de toda la vida... La EDC aspira a una participación activa y responsable del individuo en la vida democrática, a la creación de asociaciones innovadoras entre diferentes instituciones/grupos y a la equidad, la solidaridad y la cohesión social (Favinha \& Navarro Medina, 2012, pág. 5).

Ahora bien, dentro del marco de la educación ciudadana, se debe distinguir la educación formal pública o privada, de la formación que se brinda desde entidades u organismos no gubernamentales y que resultan de importancia para esta investigación, teniendo en cuenta que esta labor pretende llegar a la población que no cuenta con la posibilidad de una educación formal, o aquella que aun teniéndola, no cuenta con las herramientas para poder acceder efectivamente a los mecanismos constitucionales de protección de derechos; cobra vital importancia para esta investigación, la intervención de las instituciones de educación superior, en el área de las ciencias sociales, particularmente el Derecho y las Ciencias Políticas, considerando que cuentan con posibilidad para ofrecer la formación, capacitación y especialidad para brindar desde la pedagogía, las herramientas y las bases para que la población acceda a la información y la formación en mecanismos de protección de derechos, lo que incentiva de forma directa la participación activa de la ciudadanía en el marco de la democracia participativa dentro del Estado Social de Derecho.

Resulta oportuno expresarse frente a la función social de la Universidad, no solo entendida como la producción y réplica de conocimiento teórico, sino que debe influir de forma directa en su entorno social a través de la enseñanza de lo que Ugarte y Naval (2008-2009) han definido como competencia cívica, la cual dividen en dos:

“1. Crítico. De entre todas las competencias que facilitan la consolidación del pensamiento crítico en los estudiantes, las autoras consideran que uno de los medios más adecuados es la toma de decisiones, puesto que esta se basa en la observación, el análisis y la reflexión.

2. Participativo. Para contribuir a la mejora social, no es suficiente con adquirir y consolidar un pensamiento reflexivo y crítico. Es preciso transmitir la necesidad de participación en las cuestiones sociales y cívicas como vía para buscar respuestas a los problemas y retos sociales. La toma de decisiones puede ser una competencia que no solo facilita la reflexión 
y el análisis sino también la participación social. Ugarte y Naval (2008-2009) como se cito en (Moledo, 2012, pág. 30)

Es en este último escenario en que la universidad debe tener un papel importante en el objetivo de formar y educar a la ciudadanía, en lo que respecta a esta investigación, en los mecanismos constitucionales de garantía de los derechos, con ello incentivar un control político y la generación de políticas públicas que en definitiva tienen un impacto general en la sociedad, dado que el resultado no solo beneficia a quien logre obtener la formación y educación, si no que estará generando a través de ello un beneficio directo o indirecto a la población que se encuentra en situaciones de vulneración en casos análogos.

Teniendo en cuenta estas precisiones que dan base a la importancia de la educación, se presenta a continuación la experiencia del diplomado "Mecanismos de control y participación social: estrategias para la paz. Estudio de caso en el municipio de Zipaquirá", el cual se realizó en el segundo semestre del año 2017 por la Institución Universitaria Colegios de Colombia (UNICOC), dirigido a líderes sociales. Esta experiencia pedagógica fue desarrollada bajo los parámetros que serán presentados a continuación.

En primer lugar, surge con el objetivo de promover el uso de los mecanismos constitucionales por parte de la ciudadanía, de esta manera apoyar a la participación activa en la democracia en Colombia, bajo la pretensión de: "Fortalecer los conocimientos fundamentales de la ciudadanía que bien lo demanda la Constitución como las herramientas jurídicas, políticas y administrativas" (Moreno \& Angel, 2018 pág. 2). La metodología desarrollada integra la "acción participativa" y teoría de los campos; la primera, consiste en seleccionar una comunidad en específico, luego conocer sus problemas y necesidades, planificar métodos y destinar recursos para el buen cambio de la realidad social. Por su parte, la teoría de los campos:

\begin{abstract}
Consiste en un enfoque metodológico del constructivismo entendido como la dialéctica del sujeto investigador con los contextos, que resolviendo las problemáticas interactivas va aprendiendo y experimentado, tanto los fenómenos sociales como los núcleos problémicos que se construyen mediante el ensayo y error en las prácticas sociales. (Moreno \& Angel, 2018, pág. 11).
\end{abstract}

Ahora bien, el aporte de la metodología de Investigación Acción Participativa, I.A.P., se enfoca en que el sujeto investigado, después de haber recibido los conocimientos expuestos por parte de los docentes y con el acompañamiento de estudiantes ${ }^{5}$, tenga la capacidad de replicar los conocimientos adquiridos en su comunidad y adquiriera las competencias necesarias para enfrentar y brindar alternativas de solución a los problemas sociales de acuerdo con el contexto en el que cada uno se desenvuelve (Moreno \& Ángel, 2018,pág. 4-5).

5 En la investigación denominada "Mecanismos de control y participación social: estrategias para la paz. estudio de caso en el Municipio de Zipaquirá" participaron estudiantes del semillero de investigación del Colegio Jurídico de Unicoc. 
Para lograr el objetivo, se diseñaron conductas de entrada al inicio de cada módulo o sesión a desarrollar, para cualitativamente, establecer el nivel de conocimiento que los sujetos estudiados tenían desde su experiencia personal frente a los contenidos de formación que fueron planteados por Unicoc. Lo anterior, con el fin de determinar al final de cada sesión, el nivel de aprehensión de conocimientos que obtuvieron los sujetos, teniendo en cuenta que al finalizar cada sesión, se realiza un taller práctico con situaciones cotidianas que pueden presentarse en su entorno, en el marco de los mecanismos constitucionales de protección de derechos.

Sobre el particular, es de resaltar la calidad del sujeto investigado para el desarrollo del diplomado, en el sentido de ser considerados "líderes sociales". El nombre de líder social es un nombre que pertenece a la modernidad; el término lo explica el doctor Vicente González Radío profesor de la Universidad de la Coruña en España, en su artículo el liderazgo social:

Desde el ámbito histórico-genético hay que reseñar que el término líder aparece con la modernidad, es producto del hombre ilustrado y, de hecho, la expresión se ha generalizado siendo utilizada en el mundo anglosajón -leader-, pero se extendió a otras lenguas y empezó a tener tratamientos y significados nuevos. En ese sentido, el líder era, de forma genérica, la persona que dirige u orienta a un grupo, que reconoce su autoridad. El líder es el dirigente, el jefe. (Radío, 2006, pág. 19).

El líder de forma genérica es aquel que representa a una comunidad en cualquier ámbito; sin embargo, para desarrollar el diplomado, hicieron parte, líderes sociales que representan a sectores como la salud, que integran juntas de acción comunal, y otras que trabajan con las entidades municipales.

Es de vital importancia que en este primer ciclo se haya elegido como sujeto a investigar, líderes sociales debido a que precisamente son ellos quienes, mediante su credibilidad y confianza ciudadana, pueden replicar lo aprendido dentro del sector que representan, con el fin de fortalecer las competencias ciudadanas, la democracia y el efectivo acceso a la administración de justicia.

Por otra parte, el diplomado se dividió en 8 módulos, en los que se buscó formar a estos líderes en los distintos mecanismos de participación ciudadana que consagra la Constitución Política de 1991. Estos módulos fueron:

i. Conceptos generales de Estado, democracia, ciudadanía y paz

ii. Derechos fundamentales: ciudadanía y paz

iii. Mecanismos constitucionales de control social - mecanismos judiciales (la acción de tutela, Acción popular, acción de grupo y acciones de constitucionalidad (inconstitucionalidad, inexequibilidad y nulidad por inconstitucionalidad). 
iv. Mecanismos constitucionales de control social - mecanismos políticos (Revocatoria de Mandato, Veedurías ciudadanas, Cabildo Abierto, Referendo, Plebiscito y Consulta popular)

v. Mecanismos constitucionales de control social - mecanismos políticos administrativos

Propósito: dar a conocer los mecanismos administrativos de control social que los ciudadanos tienen a disposición para la defensa de los derechos de la comunidad.

Contenido: derecho de petición, definición y regulación por la norma colombiana, clases, términos, facultades.

En el marco de la participación que se pretendió en la investigación, el quinto modulo contó con la participación activa de los estudiantes de UNICOC, quienes hicieron parte en calidad de expositores de la correspondiente sesión. Lo anterior permitió incentivar la relación de los estudiantes y futuros profesionales con la comunidad, en este caso de Zipaquirá, Cundinamarca, que además hace parte de la zona de influencia de la universidad:

vi. empoderamiento y participación comunitaria para la promoción de la salud oral

vii. $\quad$ solución alternativa de conflictos y paz

viii. emprendimiento y finanzas personales (Moreno \& Angel, 2018, pág. 39).

Una vez expuesta de forma general la estructura del Diplomado "Mecanismos de control y participación social: estrategias para la paz. estudio de caso en el municipio de Zipaquirá", se resaltan a manera ilustrativa algunos de los resultados que a través del "Informe final resultados de investigación" en cabeza de los doctores, Álvaro Hernán Moreno Durán y Luz Karime Ángel, se presentan a la comunidad académica, en el que se logra observar, que en definitiva, este tipo de iniciativas de formación y pedagogía resultan ser de gran impacto, en este caso para una comunidad específica; así las cosas, el resultado evidenciado a través de 3 matrices que evaluaron de forma general 3 aspectos, es a grandes rasgos:

Matriz 1: Se pretendió analizar el conocimiento puntual, que se tenía frente a cada uno de los temas propuestos en los distintos módulos a través de la conducta de entrada, obteniendo como resultado: 
Los participantes en las conductas de entrada, refieren conocimientos generales, incompletos y en algunos casos tergiversados. Se denota un bajo conocimiento y muy parcializado de las categorías de análisis, especialmente en temas como Estado, ciudadanía, democracia, y aún más sobre temas tan cotidianos, como el derecho de petición.

Continúa la matriz 1 señalando:

Desarrollados los módulos, los participantes muestran la aprehensión de los temas satisfactoriamente, reflejados en los ejercicios desarrollados a través de la solución de problemas sociales, a modo de ejercicios académicos y prácticos. La conducta de salida es evaluada en términos muy positivos, en cuanto, la dedicación, el interés y la disposición de los participantes, contribuyó al cumplimiento de los objetivos del diplomado.

Visto lo anterior, es posible afirmar que si bien los sujetos al ingresar a cada sesión cuentan con conocimientos limitados o casi inexistentes frente a los temas a tratar, el desarrollo de la sesión les ha permitido asumir y apropiar los temas expuestos para aquellos que tenían algún grado de conocimiento, mientras que a quienes definitivamente no habían tenido contacto con estos mecanismos, les significó aprender y entender que existen herramientas que están a su disposición para obtener la protección de sus derechos; esto último a través de la conducta de salida, que consistió como se dijo antes, en talleres prácticos en los que se logró evidenciar la aprehensión de conocimientos y la correcta aplicación de los distintos mecanismos expuestos y desarrollados en las sesiones. (Moreno \& Angel, 2018, pág. 43) .

Matriz 2: Se pretendió entender la composición del grupo estudiado, encontrando que en efecto es un conglomerado diverso, respecto a factores, de filiación política, religiosa o cultural, ámbito de desempeño laboral y profesional, sin embargo, se reflejaron circunstancias de confluencia e interés común que radican en el liderazgo comunitario que les permite conformar grupos y colectividad en torno a temas de interés para cualquiera de sus comunidades. Se evidenció además disposición total para recibir la formación, así como una participación activa, que por momentos resultaba un diálogo sobre vivencias y experiencias propias entre los sujetos, con la posterior aclaración o explicación técnica, del expositor de cada sesión, lo cual arrojó desde el propio grupo, la iniciativa por replicar en sus comunidades los temas aprendidos en las distintas sesiones, logrando con ello el objetivo de la investigación. (Moreno \& Angel, 2018, pág. 44).

Matriz 3: Se pretendió hacer una evaluación cualitativa general del diplomado, en cuanto al plan de estudios implementado, así como la metodología utilizada, la pertinencia de los temas presentados y la aprehensión de conocimientos por parte del grupo. Los resultados arrojan un saldo favorable, pues en términos de la investigación según la 
escala utilizada de: excelente, bueno, regular e insuficiente, se ubicaron entre excelente y bueno. De este modo, recurriendo a las conclusiones de Moreno y Ángel, se: “Consolida la hipótesis, sobre la necesidad de la permanente capacitación de las comunidades, para conseguir una real participación democrática a nivel local, que con el tiempo impactará el ámbito nacional, mejorando la calidad de vida y la gobernabilidad de un Estado" (Moreno \& Angel, 2018, pág. 46).

\section{Conclusiones}

Se logra constatar, que la educación y la formación en competencias ciudadanas, específicamente en mecanismos constitucionales de protección de derechos, resulta ser una alternativa para que desde las instituciones de educación superior, se involucre a la comunidad, aportando desde la academia una formación que aun sin ser formal, resulta ser en su mayoría de gran importancia para comunidades que no tienen acceso a los contenidos, muchas veces especializados o técnicos como lo son aspectos jurídicos, políticos o administrativos, que requieren un mínimo de técnica y de rigurosidad en su presentación, en procura de lograr efectividad al momento de hacer uso de los respectivos mecanismos según sea el caso.

En este sentido y mediante el estudio de la sentencia T-760 de 2008 se logró evidenciar, en primer lugar, que a Colombia lo antecede un pasado en materia de salud difícil y que su aplicación aun hoy a pesar de diferentes mejoras legales y por las órdenes impartidas por los jueces, como la creación de la Ley 100 de 1993 y la sentencia T-760 de 2008, no es totalmente acorde con las necesidades que la sociedad presenta. Sin embargo, como se logró demostrar, efectivamente existió un control político por parte de la Corte Constitucional frente a las instituciones gubernamentales, que generaron un cambio en la administración de la salud pública, que llevaron consigo a la generación de parámetros de políticas públicas para una correcta aplicación de las órdenes dadas por la Corte Constitucional.

Siendo la anterior una sentencia de vital importancia en el sector salud y para los profesionales en Derecho, en tanto elevó la salud a la categoría de derecho fundamental, y para efectos de esta investigación, es preciso hacer énfasis en que fue en razón al uso de la acción de tutela, que se activó la potestad de la Corte Constitucional para proferir la sentencia estudiada, resultado de ello un claro ejemplo de que es posible desde la participación activa de las personas, generar control a las actuaciones de la administración en cualquiera de sus niveles y aportar e influir en la creación o actualización de políticas públicas, que en efecto respondan a las necesidades que desde la sociedad se evidencian.

Finalmente, en el marco de la demostración de la importancia que tiene y debe tener la educación y la formación de la ciudadanía, en procura de lograr un empoderamiento en 
cuanto a la existencia y la aplicación de los mecanismos constitucionales de protección de derechos, se deja planteada la alternativa pedagógica a manera iniciativa institucional desde Unicoc, frente a la creación de diplomados dirigidos a poblaciones específicas, como lo son los líderes sociales que, cuentan con cualidades como el reconocimiento social y comunitario importante, que los reviste de credibilidad y capacidad de consenso y unión en las comunidades, lo cual se pretende lograr a través de la formación por sesiones o módulos de los distintos mecanismos que prevé la Constitución Política de Colombia y que aun estando al alcance de todos, se demostró que la gran mayoría de la población objeto de estudio como muestra de la sociedad colombiana, no conoce los distintos mecanismos, o si bien los conocen, tiene serias distorsiones entre sus conocimientos y los procedimientos, términos y rigurosidades que se exigen en cada uno. Es por ello entonces que este tipo de alternativas formativas, resultan además ser innovadoras y de utilidad considerable, en procura de incentivar y fomentar el aprendizaje y posteriormente replicar en las comunidades, los conocimientos que se adquieren a lo largo del diplomado, que en definitiva crea lazos y agrupaciones comunitarias, permitiendo que cada vez sea más la población que pueda tener acceso y que cuente con las herramientas necesarias para por sus medios, acudir o interponer las distintas acciones o mecanismos constitucionales que existen en la Constitución Nacional, pero que en muchos casos les es de difícil acceso.

\section{Referencias bibliográficas}

Castrillon, J. J. (2013). El sistema de salud en Colombia. Archivos de Medicina , 115-116. Corte Constitucional, Sentencia T-760-2008 (Corte Contitucional 31 de julio de 2008).

Favinha, M., \& Navarro Medina, E. (2012). La importancia de la formación ciudadana en la educación vista. Actas do II Congresso Internacional, Interfaces da Psicologia: Qualidade de Vida-Vidas de Qualidade, (pág. 17).

Henao, J.(2004). Panorama del Derecho Constitucional Colombiano. Bogota: Temis S.A. Congreso de Colombia. (8 de febrero de 1994). Ley General de Educación. (Ley 115 de 1194).

Consejo Nacional Legislativo, Colombia ( 20 de octubre de 1886). Ley que crea las Juntas de Higiene en la Capital de la República y en los Departamentos y Ciudades principales. (Ley 30 de 1886)

Moledo, M. L. (2012). UNIRIOJA. Obtenido de UNIRIOJA: https://dialnet.unirioja.es/ descarga/articulo/4147392.pdf 
Moreno, A, y Ángel, L (2018). Informe final resultados de investigación: Mecanismos de control y participación social: Estrategias para la paz. Estudio de caso en el municipio de Zipaquirá.

Murillo Castaño , G., \& Castañeda , N. (2007). Competencias ciudadanas y construcción de ciudadanía juvenil . Reforma y Democracia, 18.

Neiva, P. d. (s.f.). Personeria de Neiva. Recuperado el 10 de 04 de 2018, de http://www. personerianeiva.gov.co/index.php/mecanismos-constitucionales-para-la-proteccion-de-los-derechos-humanos

Pueblo, D. d. (2009). La tutela yel derecho a la salud periodo 2006 a 2008. Cundinamarca. Bogotá: Defensoría del pueblo.

Quinche, M. F., \& Rivera, J. C. (2010). El control judicial de las políticas. Vniversitas, 137.

Radio, V. G. (2006). El liderazgo social. Revista galego-portuguesa de psicoloxía e educación, 19.

Rincón. (2013). El control politico en Colombia. Derecho y realidad, 361.

Ruiz Silva, A., \& Chaux Torres, E. (2005). La formuacion de competencias ciudadanas. ASOFADE, 2-100.

Salud, M. d. (s.f.). Gobierno de Colombia. Recuperado el 10 de 04 de 2018, de https:// www.minsalud.gov.co/proteccionsocial/Paginas/Sistema-general-de-Pensiones. aspx

Salud, M. d. (s.f.). Gobierno de Colombia. Recuperado el 10 de 04 de 2018, de https:// www.minsalud.gov.co/proteccionsocial/RiesgosLaborales/Paginas/afiliacion-sistema-general-riesgos-laborales.aspx

Salud, M. d. (s.f.). Ministerio de salud. Recuperado el 10 de 04 de 2018, de https://www. minsalud.gov.co/proteccionsocial/Paginas/pos.aspx

Salud, M. d. (s.f.). Ministerio de Salud. Recuperado el 10 de 04 de 2018, de https:// www.minsalud.gov.co/Lists/Glosario/DispForm.aspx?ID=133\&ContentTypel$\mathrm{d}=0 \times 0100 B 5 A 58125280$ A70438C125863FF136F22

Social, M. d. (2004). Ministerio de la Protección Social. Recuperado el 10 de 04 de 2018, de Sistema de Seguridad Social en Salud: https://www.minsalud.gov. co/Documentos\%20y\%20Publicaciones/GUIA\%20INFORMATIVA\%20DEL\%20 REGIMEN\%20CONTRIBUTIVO.pdf. 
Valencia, M., Borrero, Y. E., Pérez, E. M., Muñoz, N. E., \& Cáceres, F. (25 de septiembre de 2009). Dialnet. Recuperado el 9 de Abril de 2018, de Modificaciones al Sistema General de Seguridad en Salud a la luz de una sentencia de la Corte constitucional : file://C:/Users/beltran/Downloads/Dialnet-ModificacionesAlSistemaGeneralDeSeguridadSocialEnS-3987432.pdf. 